\title{
Blind Identification of FIR Channels with Multiple Users via Spatio-Temporal Processing
}

\author{
H. Aghajan, B. Hassibi, B. Khalaj, A. Paulraj, and T. Kailath \\ Information Systems Laboratory, Department of Electrical Engineering \\ Stanford University, Stanford, CA 94305, USA
}

\begin{abstract}
A new method is proposed for blind identification of possibly nonminimum phase FIR channels with multiple users. The technique exploits the structure of the signals received by an antenna array in both the temporal and spatial frequency domains. Although in the single antenna case it is necessary to use cyclostationary signals or higher order statistics to identify the magnitude and phase of the channel, we circumvent such a requirement by exploiting certain multichannel features of the array. We show that if multiple users are present, the nonminimum phase channels associated with each user can still be identified from the second-order statistics, provided additional spatial structure exists.
\end{abstract}

\section{Introduction}

Equalization of a communications channel requires implicit or explicit knowledge of its transfer function. A communication channel is usually identified by LMS or RLStype adaptive algorithms in which the reference signal is provided by transmitting known training sequences. The so-called blind channel identification techniques only use the channel output and some known statistical properties of the transmitted signal. As a result, these techniques have the potential to increase the transmission capability by eliminating training sequences.

It is well known that nonminimum phase channels driven by wide-sense stationary input sequences, cannot be identified from second-order statistics. Therefore blind identification techniques, to date, use either higher order statistics $[1,2,3,4]$ or use cyclostationary input signals $[5,6,7]$ to identify a possibly nonminimum phase channel. Due to their slow rate of convergence, these techniques may be impractical for mobile communications environments.

In general, signals arrive at the receiver not only with different delays, but also from different spatial angles. In digital transmission systems, antenna arrays have recently attracted much attention in the framework of optimal spatial diversity combining $[7,8]$. In [9] it was shown that for the antenna array case, second-order statistics provide enough information for identifying the channel, and a method was proposed on this basis.

In the multiple user case, second-order statistics are not sufficient to uniquely identify the channels for each user. Additional information or structure has to be exploited in order to obtain unique estimates of all the channel coefficients. In this paper, we attempt to use the spatial structure of the incoming signals. We shall therefore introduce a two-dimensional channel concept, which is a generalization of the usual one-dimensional channel in the following sense.

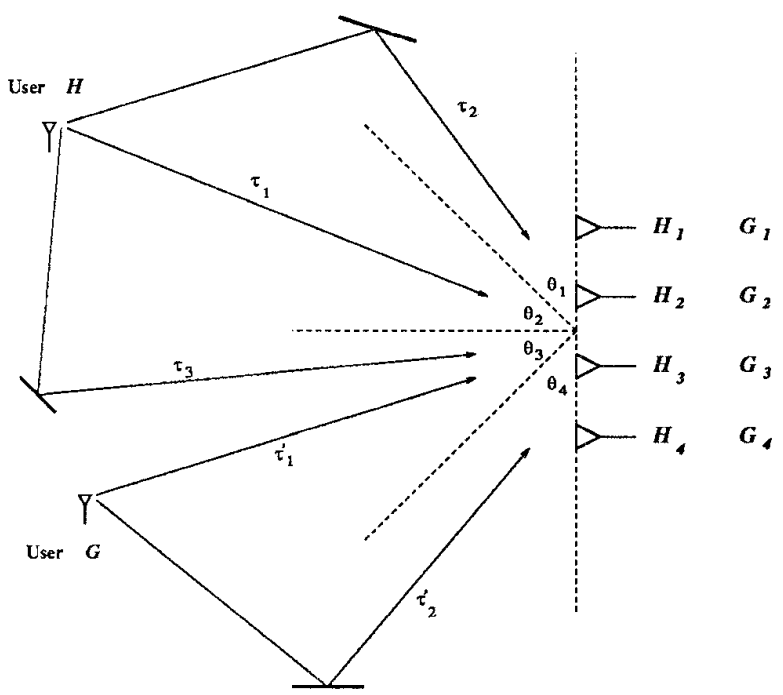

Figure 1: A typical multi-user channel model

A receiving system typically receives signals from each user via different paths that combine and introduce InterSymbol-Interference in the measurements. Figure 1 shows a typical propagation model, in which four antenna elements receive signals from two users via multiple paths. The channel can hence be characterized in the time domain by an FIR filter with coefficients related to the ISI strengths. On the other hand, one can view the channel in the spatial frequency domain as the summation of contributions from multiple paths distributed in the spatial frequency space. In other words, if multiple antenna elements are used in the receiving system, then one can characterize the channel by considering the spatial correlation of the received snapshots, ignoring temporal structure. For example, the antenna elements in Figure 1 effectively partition the spatial frequency axis $(\theta)$ into four sectors. By exploiting both the temporal and spatial structures in the measurements, one can expect improved performance in the channel iden- 


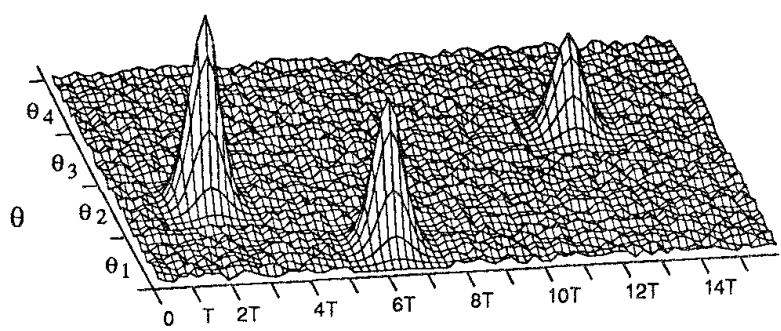

$\tau$

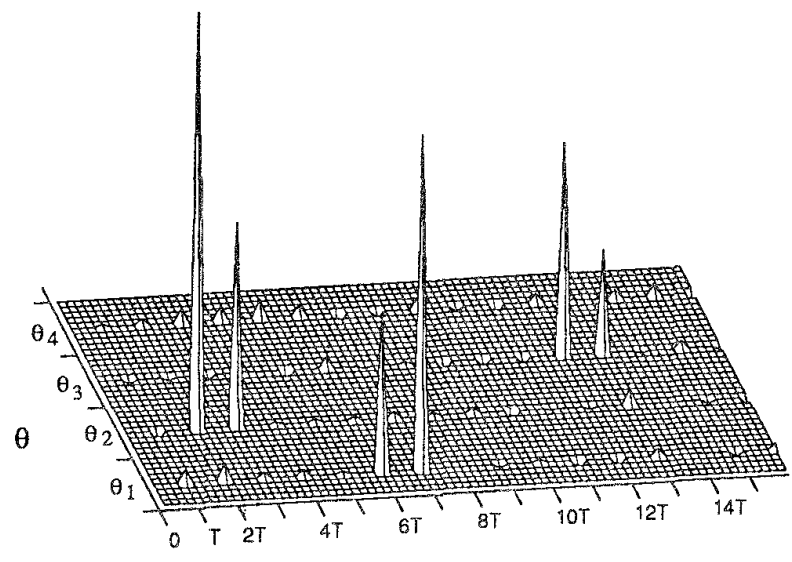

$\tau$

Figure 2: 2-D spatio-temporal continuous and discrete channels for user $H$.

tification system. The channel can therefore be regarded as an entity in a two-dimensional space with temporal and spatial axes. One advantage of this formulation is the improved resolvability of the correlation peaks in cases where either of the two sampling rates (in time or space) severely limits the resolution. Figure 2 visualizes such 2-D channel models in the continuous and discrete time. The algorithm presented in this paper is an attempt to exploit both the spatial and temporal characteristics of the channel. Our analysis will show that in order to identify the channels for all $d$ users, at least $d+1$ antenna elements are needed.

The signals impinging upon the antenna array from the different users arrive as planar waves at different angles and different delays. We shall assume that for each user there exists some direction in which that user's contribution is dominant. As will be shown later, under this assumption we can identify the channels corresponding to each user. In our analysis, we consider a uniform linear array of sensors. However, the technique is applicable to any known array structure.

The algorithm is based on the evaluation of the power spectrum matrix of the measurements in both the temporal and spatial frequency domains. To obtain this spectrum $S_{\theta}(z)$, we first compute the spatial frequency transform of the measurements $x(t)$ and obtain a vector function of time, $\Theta(t)$. Then, the autocorrelation matrix, $R_{\theta}(\tau)$, of $\Theta(t)$ is computed. Finally, the matrix power spectrum of the array output, $S_{\theta}(z)$, is computed as the $z$-transform of $R_{\theta}(\tau)$.

\section{The Data Model}

We shall presently consider the case for two users; generalizing the method to $d$ users is straightforward.

In a PAM data communication system the baseband transmitted signals $u_{1}(t)$ and $u_{2}(t)$ are given by

$$
u_{j}(t)=\sum_{k=-\infty}^{\infty} s_{k}^{j} p(t-k T) \quad j=1,2
$$

where $T$ is the symbol period, $p(\cdot)$ is the pulse shaping function, and $s_{k}^{j}$ is the transmitted sequence of user $j$.

The signal received at the $i$-th antenna element is then

$$
x_{i}(t)=h_{i}^{c}(t) \otimes u_{1}(t)+g_{i}^{c}(t) \otimes u_{2}(t)+n_{i}(t)
$$

where $h_{i}^{c}(t)$ and $g_{i}^{c}(t)$ are the channel impulse responses of the $i$-th antenna, and $n_{i}(t)$ is the corresponding additive noise process. By combining pulse shaping and "channel impulse response functions in composite channel responses $h_{i}(t)$ and $g_{i}(t)$, we get

$$
x_{i}(t)=\sum_{k=-\infty}^{\infty} s_{k}^{1} h_{i}(t-k T)+\sum_{k=-\infty}^{\infty} s_{k}^{2} g_{i}(t-k T)+n_{i}(t)
$$

The output of an antenna array with $M$ elements can be written in vector form as

$$
\mathrm{x}(t)=\left[x_{1}(t), x_{2}(t), \ldots, x_{M}(t)\right]^{T} .
$$

In our model we will make the following assumptions:

1. The channel impulse responses $h_{i}(t)$ and $g_{i}(t)$ are assumed to be finite impulse response (FIR) filters.

2. The noise terms $n_{i}(t)$ are zero-mean, uncorrelated with $s_{k}^{j}, j=1,2$, and white in time and space.

$$
E\left[n_{i}\left(t_{1}\right) n_{j}\left(t_{2}\right)\right]=\left\{\begin{array}{cc}
\sigma^{2} & \text { when } i=j \text { and } t_{1}=t_{2} \\
0 & \text { otherwise }
\end{array}\right.
$$

We shall assume $\sigma^{2}$ to be known, although this condition may be relaxed. 
3. For convenience, the transmitted sequences $s_{k}^{j}, j=1,2$ are assumed to be zero-mean and iid, $E\left[s_{k}^{j} s_{l}^{j}\right]=\delta(k-$ $l), j=1,2$. The algorithm still works for a general input with known power spectral density as long as the users are independent.

\section{The Multichannel Identification Approach}

Denote the autocorrelation of $\mathbf{x}(t)$ by

$$
R_{\mathbf{x}}(t, \tau)=E\left[\mathbf{x}(t) \mathbf{x}^{*}(t-\tau)\right]
$$

By sampling the output signal $\mathbf{x}(t)$ at the symbol rate $1 / T$, the discrete signal, $\mathbf{x}[k]=\mathbf{x}(k T)$, will be a wide-sense stationary signal with autocorrelation function

$$
R_{\mathbf{x}}[m]=E\left(\mathbf{x}[k] \mathbf{x}^{*}[k-m]\right)
$$

The elements of $R_{\mathbf{x}}[m]$ can be written as

$$
\begin{aligned}
R_{x_{i} x_{j}}[m] & =E\left(\mathbf{x}_{i}[k] \mathbf{x}_{j}^{*}[k-m]\right) \\
& =E\left(\left(s_{l}^{1}\right)^{2}\right) h_{i}[m] \otimes h_{j}^{*}[-m] \\
& +E\left(\left(s_{l}^{2}\right)^{2}\right) g_{i}[m] \otimes g_{j}^{*}[-m]+\sigma^{2} \delta[m] \delta[i-j](8)
\end{aligned}
$$

where $h_{i}[k]=h_{i}(k T)$. The power spectrum $S_{\mathrm{x}}(z)$, defined to be the $z$-transform of $R_{\mathbf{x}}[m]$, is then given by for any unitary matrix $\mathrm{U}$.

We now look at this problem in the spatial frequency domain. To do so, we first calculate the spatial frequency transform of the measurement vector $\mathbf{x}(t)$

$$
\Theta(t)=W_{\mathbf{x}}(t)
$$

where $\mathbf{W}$ is the array response matrix at say $M$ different spatial angles. This leads to the following quantities

$$
\begin{aligned}
S_{\Theta}(z) & =\mathbf{W} S_{\mathbf{x}}(z) \mathbf{W}^{*} \\
& =\left[\begin{array}{ll}
\mathbf{H}_{\theta}(z) & \mathbf{G}_{\theta}(z)
\end{array}\right]\left[\begin{array}{l}
\mathbf{H}_{\theta}{ }^{*}\left(z^{-1}\right) \\
\mathbf{G}_{\theta}^{*}\left(z^{-1}\right)
\end{array}\right]+\sigma^{2} I
\end{aligned}
$$

where we have defined

$$
\left[\begin{array}{ll}
\mathbf{H}_{\theta}(z) & \mathbf{G}_{\theta}(z)
\end{array}\right]=\mathbf{W}\left[\begin{array}{ll}
\mathbf{H}(z) & \mathbf{G}(z)
\end{array}\right]
$$

Assumption 1: For each user there exists a direction (spatial frequency bin) where that user's contribution is dominant. In other words, the power received from other users in this spatial frequency bin is negligible compared to the power received from that user.

$$
S_{\mathbf{x}}(z)=\mathcal{Z}\left(R_{\mathbf{x}}[m]\right)=\left(\begin{array}{ccc}
H_{1}(z) H_{1}^{*}\left(z^{-1}\right)+G_{1}(z) G_{1}^{*}\left(z^{-1}\right) & \ldots & H_{1}(z) H_{M}^{*}\left(z^{-1}\right)+G_{1}(z) G_{M}^{*}\left(z^{-1}\right) \\
H_{2}(z) H_{1}^{*}\left(z^{-1}\right)+G_{2}(z) G_{1}^{*}\left(z^{-1}\right) & \ldots & H_{2}(z) H_{M}^{*}\left(z^{-1}\right)+G_{2}(z) G_{M}^{*}\left(z^{-1}\right) \\
\vdots & \ddots & \vdots \\
H_{M}(z) H_{1}^{*}\left(z^{-1}\right)+G_{M}(z) G_{1}^{*}\left(z^{-1}\right) & \ldots & H_{M}(z) H_{M}^{*}\left(z^{-1}\right)+G_{M}(z) G_{M}^{*}\left(z^{-1}\right)
\end{array}\right)+\sigma^{2} I
$$

which can be written as

$$
S_{\mathbf{x}}(z)=\left[\begin{array}{ll}
\mathbf{H}(z) & \mathbf{G}(z)
\end{array}\right]\left[\begin{array}{l}
\mathbf{H}^{*}\left(z^{-1}\right) \\
\mathbf{G}^{*}\left(z^{-1}\right)
\end{array}\right]+\sigma^{2} I
$$

where

$$
\begin{aligned}
\mathbf{H}(z) & =\left[H_{1}(z), H_{2}(z), \ldots, H_{M}(z)\right]^{T} \\
\mathbf{G}(z) & =\left[G_{1}(z), G_{2}(z), \ldots, G_{M}(z)\right]^{T}
\end{aligned}
$$

are the multichannel transfer function vectors.

Note that although $R_{\mathbf{x}}(\tau)$ has full rank, in the noise free case Eq. (10) shows that $S_{\mathbf{x}}(z)$ will have rank two. This is independent of the number of multipaths present, and will be crucial for identifying the channels.

In the single user case $\mathrm{G}(z) \equiv 0$, and the channels in $\mathrm{H}(z)$ can be identified from (10) using the method of [9]. However, in the multiple user case $\mathbf{H}(z)$ and $\mathbf{G}(z)$ cannot be uniquely identified from (10), since if $\overline{\mathbf{H}}(z)$ and $\overline{\mathbf{G}}(z)$ satisfy $(10)$, then so will

$$
\left[\begin{array}{ll}
\mathbf{H}(z) & \mathrm{G}(z)
\end{array}\right]=\left[\begin{array}{ll}
\overline{\mathbf{H}}(z) & \overline{\mathbf{G}}(z)
\end{array}\right] \mathbf{U}
$$

The above assumption requires that the channel response vectors $\mathbf{H}_{\theta}(z)$ and $\mathbf{G}_{\theta}(z)$ have the following form

$$
\left[\begin{array}{ll}
\mathbf{H}_{\theta}(z) & \mathrm{G}_{\theta}(z)
\end{array}\right]=\left[\begin{array}{cc}
\times & \times \\
\vdots & \vdots \\
\times & 0 \\
\vdots & \vdots \\
0 & \times \\
\vdots & \vdots \\
\times & \times
\end{array}\right] \leftarrow \theta_{i}
$$

In other words, user 1 is dominant in spatial frequency bin $\theta_{i}$, and user 2 is dominant in $\theta_{j}$. It is readily observed from (18) that the only unitary matrix that will preserve the structure of the channel response vectors is 


$$
U=\left[\begin{array}{cc}
e^{j \Omega_{1}} & 0 \\
0 & e^{j \Omega_{2}}
\end{array}\right]
$$

Therefore, the channels are uniquely identifiable up to such a constant phase factor. We can thus show the following.

\section{Multichannel Identifiability Condition for Multiple Users}

If Assumption 1 is satisfied, then the $2 M$ channels $h_{i}[n]$ and $g_{i}[n]$ are uniquely identifiable up to a constant phase factor if, and only if, the channel transfer functions $\left\{H_{\theta_{i}}(z)\right\}_{i=1}^{M}$ have no common roots and the channel transfer functions $\left\{G_{\theta_{i}}(z)\right\}_{i=1}^{M}$ have no common roots.

Example: Suppose we have an antenna array with $M=3$ elements that receive signals from two users. User 1 has contributions in the spatial frequency bins $\theta_{1}$ and $\theta_{2}$, and user 2 has contributions in the bins $\theta_{1}$ and $\theta_{3}$. Therefore, user 1 is dominant in $\theta_{2}$ and user 2 is dominant in $\theta_{3} . S_{\theta}(z)$ will thus have the following form

$$
\begin{aligned}
& S_{\theta_{i} \theta_{i}}(z)=H_{\theta_{i}}(z) H_{\theta_{i}}^{*}\left(z^{-1}\right)=\sum_{l=-L}^{L} S_{\theta_{i} \theta_{i}}^{(l)} z^{l} \\
& S_{\theta_{i} \theta_{k}}(z)=H_{\theta_{i}}(z) H_{\theta_{k}}^{*}\left(z^{-1}\right)=\sum_{l=-L}^{L} S_{\theta_{i} \theta_{k}}^{(l)} z^{l}
\end{aligned}
$$

for $k=1,2, \ldots, M . L$ is the length of the channel. Now

$$
S_{\theta_{i} \theta_{i}}(z) H_{\theta_{k}}^{*}\left(z^{-1}\right)-S_{\theta_{i} \theta_{k}}(z) H_{\theta_{i}}^{*}\left(z^{-1}\right)=0
$$

for $k=1,2, \ldots, M$. Equating coefficients of the powers of $z$ in (23) yields the matrix equation

$$
\mathcal{S}\left(S_{\theta_{i} \theta_{i}},-S_{\theta_{i} \theta_{k}}\right)\left[\begin{array}{c}
h_{\theta_{k}, L}^{*} \\
\vdots \\
h_{\theta_{k}, 0}^{*} \\
h_{\theta_{i}, L}^{*} \\
\vdots \\
h_{\theta_{i}, 0}^{*}
\end{array}\right]=0, k=1,2, \ldots, M
$$

$S_{\theta}(z)=\left(\begin{array}{ccc}H_{\theta_{1}}(z) H_{\theta_{1}}^{*}\left(z^{-1}\right)+G_{\theta_{1}}(z) G_{\theta_{1}}^{*}\left(z^{-1}\right) & H_{\theta_{1}}(z) H_{\theta_{2}}^{*}\left(z^{-1}\right) & G_{\theta_{1}}(z) G_{\theta_{3}}^{*}\left(z^{-1}\right) \\ H_{\theta_{2}}(z) H_{\theta_{1}}^{*}\left(z^{-1}\right) & H_{\theta_{2}}(z) H_{\theta_{2}}^{*}\left(z^{-1}\right) & 0 \\ G_{\theta_{3}}(z) G_{\theta_{1}}^{*}\left(z^{-1}\right) & 0 & G_{\theta_{3}}(z) G_{\theta_{3}}^{*}\left(z^{-1}\right)\end{array}\right)$.

By computing $S_{\theta}(z)$ we can deduce the spatial configuration of the received signals and identify each channel. The fact that the $(2,3)$ element of $S_{\theta}(z)$ is zero indicates that there is no user contributing to both $\theta_{2}$ and $\theta_{3}$. Thus user 1 is dominant in $\theta_{2}$ and user 2 is dominant in $\theta_{3}$. Once we have made this association, we may identify $G_{\theta_{1}}(z)$ and $G_{\theta_{3}}(z)$ by finding the common factor between the polynomials $G_{\theta_{3}}(z) G_{\theta_{1}}^{*}\left(z^{-1}\right)$ and $G_{\theta_{3}}(z) G_{\theta_{3}}^{*}\left(z^{-1}\right)$. Likewise, $H_{\theta_{1}}(z)$ and $H_{\theta_{2}}(z)$ can be identified by finding the common factor between the polynomials $H_{\theta_{2}}(z) H_{\theta_{1}}^{*}\left(z^{-1}\right)$ and $H_{\theta_{2}}(z) H_{\theta_{2}}^{*}\left(z^{-1}\right)$

In what follows, we shall present a robust method for finding the common factors of polynomials that is based upon computing the smallest singular vector of a certain generalized Sylvester resultant matrix.

\section{The Channel Identification Algorithm}

In this section we shall present a method for identifying the channels $\left\{H_{\theta_{i}}(z)\right\}_{i=1}^{M}$ and $\left\{G_{\theta_{i}}(z)\right\}_{i=1}^{M}$ from the power spectrum matrix $S_{\theta}(z)$. Here we only show the procedure of identifying the $\left\{H_{\theta_{i}}(z)\right\}_{i=1}^{M}$; a similar treatment is applicable to the identification of $\left\{G_{\theta_{i}}(z)\right\}_{i=1}^{M}$. We shall assume that we know (or have estimated) the noise covariance $\sigma^{2} I$, and have subtracted it out of $S_{\mathbf{x}}(z)$. Suppose user 1 is dominant in the $i$-th frequency bin, and therefore consider the $i$-th row of $S_{\theta}(z)$ : where

$$
H_{\theta_{i}}(z)=\sum_{l=0}^{L} h_{\theta_{i}, l} z^{l}, \quad H_{\theta_{k}}(z)=\sum_{l=0}^{L} h_{\theta_{k}, l} z^{l},
$$

and $\mathcal{S}(a, b)$ is the $(3 L+1) \times(2 L+2)$ Sylvester matrix

$$
\mathcal{S}(a, b)=\left[\begin{array}{cccccccc}
a_{0} & & & & b_{0} & & & \\
a_{1} & a_{0} & & & b_{1} & b_{0} & & \\
\vdots & a_{1} & \ddots & & \vdots & b_{1} & \ddots & \\
a_{2 L} & \vdots & \ddots & a_{0} & b_{2 L} & \vdots & \ddots & b_{0} \\
& a_{2 L} & & a_{1} & & b_{2 L} & & b_{1} \\
& & \ddots & \vdots & & & \ddots & \vdots \\
& & & a_{2 L} & & & & b_{2 L}
\end{array}\right]
$$

with $a(z)=\sum_{l=-L}^{L} a_{L+l} z^{l}, b(z)=\sum_{l=-L}^{L} b_{L+l} z^{l}$. We can now prove the following result.

Theorem: The channels $\left\{H_{\theta_{i}}(z)\right\}_{i=1}^{M}$ and $\left\{H_{\theta_{k}}(z)\right\}_{i=1}^{M}$ are uniquely identifiable if, and only if, $\mathcal{S}_{i, k} \triangleq \mathcal{S}\left(S_{\theta_{i} \theta_{i}},-S_{\theta_{i} \theta_{k}}\right)$ has a nullspace of dimension one. In this case the coefficients of the $i$-th and $k$-th channels are given by solving (24). 
This theorem is of particular interest since it relates channel identifiability to a power spectrum, which we can estimate, and because it motivates the following algorithm for channel identification.

In practice we can only estimate the power spectrum from a finite number of samples. Hence

$$
\hat{S}_{\theta_{2} \theta_{i}}(z) H_{\theta_{k}}^{*}\left(z^{-1}\right)-\hat{S}_{\theta_{i} \theta_{k}}(z) H_{\theta_{i}}^{*}\left(z^{-1}\right) \neq 0
$$

for $k=1,2, \ldots, M$, where the caret denotes estimates of the spectrum. To estimate the channels, we propose

$$
\min _{H_{\theta_{i}}^{*}\left(z^{-1}\right), H_{\theta_{k}}^{*}\left(z^{-1}\right) \neq 0} \begin{aligned}
& \sum_{k=0}^{M} \| \hat{S}_{\theta_{i} \theta_{i}}(z) H_{\theta_{k}}^{*}\left(z^{-1}\right) \\
& -\hat{S}_{\theta_{i} \theta_{k}}(z) H_{\theta_{i}}^{*}\left(z^{-1}\right) \|_{2}^{2}
\end{aligned}
$$

The solution is given by the smallest right singular vector of $\hat{\mathcal{S}}_{i, k}$. We thus have the following algorithm.

\section{The Algorithm}

- Compute the spatial frequency transform of $\mathbf{x}(t)$ :

$$
\Theta(t)=\mathbf{W} \mathbf{x}(t)
$$

- Calculate an estimate of the power spectrum $\hat{S}_{\theta}(z)$.

- Use the structure of $S_{\theta}(z)$ to associate the users to different spatial frequency bins (as in the example).

- Form $\hat{\mathcal{S}}_{i, k}$, where $\theta_{i}$ is a direction in which a user is dominant, and $\theta_{k}$ is another direction in which the user has some contribution.

- Calculate the smallest right singular vector of $\hat{\mathcal{S}}_{i, k}$ to obtain estimates of the coefficients of $H_{\theta_{i}}(z)$ and $H_{\theta_{k}}(z)$.

\section{Concluding Remarks}

A new method for spatio-temporal blind identification of FIR channels for multiple users is proposed, using second order statistics and based on exploiting the multichannel structure of the system and the spatial structure of the incoming signals. The channel in the proposed framework is regarded as a generalization of the usual one-dimensional channel, and is considered as a two-dimensional function of both the time and the spatial frequency.

\section{Acknowledgments}

This work was supported in part by the Advanced Research Projects Agency of the Department of Defense and was monitored by the Air Force Office of Scientific Research under contract F49620-93-1-0085. (The United States Government is authorized to reproduce and distribute reprints for governmental purposes notwithstanding any copyright notation hereon). The views and conclusions contained in this document are those of the authors and should not be interpreted as necessarily representing the official policies or endorsements, either expressed or implied, of the Air Force Office of Scientific Research or the U.S. Government. Support was also provided by the Army Research Office under grant DAAH04-93-G-0029.

\section{References}

[1] J.K. Tugnait, "Identification of linear stochastic systems via second and fourth-order cumulant matching", IEEE Transactions on Information Theory, 33(3):393407, May 1987.

[2] O. Shalvi and E. Weinstein, "New criteria for blind deconvolution of nonminimum phase systems", IEEE Transactions on Information Theory, 36(2):312-320, March 1990.

[3] B. Porat and B. Friedlander, "Blind equalization of digital communication channels using high-order moments", IEEE Transactions on Signal Processing, 39(2):522$526,1991$.

[4] D. Hatzinakos and C. Nikias, "Estimation of multipath channel response in frequency selective channels", IEEE J. Select. Areas Commun., 7(1):12-19, January 1989.

[5] W. Gardner, "A new method of channel identification", IEEE Transactions on Communications, 39(6):813817, June 1991.

[6] L. Tong, G. Xu, and T. Kailath, "A new approach to blind identification and equalization of multipath channels", In Proc. of 25th Asilomar Conference on Circuits, Systems and Comp., 1991.

[7] L. Tong, G. Xu, B. Hassibi, and T. Kailath, "Blind identification and equalization of multipath channels: A Frequency domain approach", submitted to IEEE Transactions on Information Theory.

[8] P. Balaban and J. Salz, "Optimum Diversity Combining and Equalization in Digital Data Transmission with Applications to Cellular Mobile Radio - Part I: Theoretical Considerations", IEEE Transactions on Communications, 40(5):885-894, May 1992.

[9] B. Khalaj, B. Hassibi, A. Paulraj, and T. Kailath, "Blind identification of FIR channels via antenna arrays", In Proc. of 27 Asilomar Conf. on Circuits, Systems and Computers, 1993. 